\title{
Transcutaneous spinal stimulation as a therapeutic strategy for spinal cord injury: state of the art
}

\author{
This article was published in the following Dove Press journal: \\ Journal of Neurorestoratology \\ 23 March 2015 \\ Number of times this article has been viewed
}

\section{Leandro H Grecco $1,3,4, *$ \\ Shasha $\mathrm{Li}^{1,5, *}$ \\ Sarah Michel ${ }^{1,6, *}$ \\ Laura Castillo-Saavedra' \\ Andoni Mourdoukoutas ${ }^{7}$ \\ Marom Bikson ${ }^{7}$ \\ Felipe Fregni ${ }^{1,2}$}

'Spaulding Neuromodulation Center, Spaulding Rehabilitation Hospital, Harvard Medical School, Boston, MA, ${ }^{2}$ Spaulding-Harvard Spinal Cord Injury Model System, Spaulding Rehabilitation Hospital, Harvard Medical School, Charlestown, MA, USA; ${ }^{3}$ Special Laboratory of Pain and Signaling, Butantan Institute, ${ }^{4}$ Department of Pharmacology, Institute of Biomedical Science, University of São Paulo, São Paulo, Brazil; ${ }^{5}$ Department of Rehabilitation Medicine, West China Hospital, Sichuan University, Chengdu, Sichuan, People's Republic of China; ${ }^{6}$ Department of Pharmacy and Biomedical Sciences, University of Namur, Belgium; ${ }^{7}$ Department of Biomedical Engineering, The City College of New York, New York, NY, USA

*These authors contributed equally to this work
Correspondence: Felipe Fregni Spaulding Neuromodulation Center, Spaulding Rehabilitation Hospital, Harvard Medical School, 79/96 I3th Street, Charlestown, MA 02129, USA

Email felipe.fregni@ppcr.hms.harvard.edu

\begin{abstract}
Treatments for spinal cord injury (SCI) still have limited effects. Electrical stimulation might facilitate plastic changes in affected spinal circuitries that may be beneficial in improving motor function and spasticity or SCI-related neuropathic pain. Based on available animal and clinical evidence, we critically reviewed the physiological basis and therapeutic action of transcutaneous spinal cord stimulation in SCI. We analyzed the literature published on PubMed to date, looking for the role of three main noninvasive stimulation techniques in the recovery process of SCI and focusing mainly on transcutaneous spinal stimulation. This review discusses the main clinical applications, latest advances, and limitations of noninvasive electrical stimulation of the spinal cord. Although most recent research in this topic has focused on transcutaneous spinal direct current stimulation (tsDCS), we also reviewed the technique of transcutaneous electric nerve stimulation (TENS) and neuromuscular electrical stimulation (NMES) as potential methods to modulate spinal cord plasticity. We also developed a finite element method (FEM) model to predict current flow in the spinal cord when using different electrode montages. We identified gaps in our knowledge of noninvasive electrical stimulation in the modulation of spinal neuronal networks in patients with SCI. tsDCS, TENS, and NMES have a positive influence on the promotion of plasticity in SCI. Although there are no randomized controlled studies of tsDCS in SCI, preliminary evidence is encouraging. FEMs predict that tsDCS electrode montage can be used to shape which spinal segments are modulated and what detailed areas of spinal anatomy can concentrate current density (eg, spinal roots). tsDCS is a technique that can influence conduction along ascending tracts in the spinal cord, so could modulate supraspinal activity. It may also be a promising new approach for a number of neuropsychiatric conditions.
\end{abstract}

Keywords: spinal cord injury, transcutaneous spinal direct current stimulation, transcutaneous electric nerve stimulation, neuromuscular electrical stimulation, motor, spasticity, pain

\section{Introduction}

The global annual incidence of spinal cord injury (SCI) is 22 cases per million people, according to a report published in 2007, indicating that there are approximately 2.5 million people currently living with SCI. ${ }^{1}$ Up to 2011 , the prevalence of SCI in the USA ranged from 721 to 1,009 per million, and the annual incidence was estimated to range between 11.5 and 53.4 per million people. ${ }^{2}$ Patients surviving an SCI usually experience prolonged hospitalization in acute care units and rehabilitation centers. Although different therapeutic strategies in the past have focused on improving individual post-injury management, ${ }^{3,4}$ there is a growing need for new treatment strategies to ameliorate recovery of function in these patients. One way to achieve an optimal treatment approach is to take advantage of the intrinsic ability of the spinal circuits to 
remodel and reconstruct the remaining pathways, ${ }^{5,6}$ which are kept intact but preserve a subthreshold level for evoking motor neuronal responses, and could drive spinal plasticity in SCI patients.

It has been established that after a spinal cord lesion, the neural circuitry below the injury undergoes substantial modifications in segmental connections due to the dramatic loss of supraspinal and propriospinal inputs. The loss or disruption of descending input results in substantial changes at the level of the primary afferents, interneurons, and motor neurons, thus significantly affecting connectivity in the spinal cord. ${ }^{7}$ The spinal cord has extraordinary potential for neuroplasticity, ${ }^{1}$ so electrical stimulation could enhance and facilitate plastic changes in the affected spinal circuitries, leading to an improvement in functional motor performance, ${ }^{8}$ and aid in the treatment of neuropathic pain in SCI. ${ }^{9}$

This comprehensive review provides an overview of the physiological basis and therapeutic action of transcutaneous spinal stimulation in SCI. It focuses on available animal and clinical evidence, the main clinical applications, and latest advances and limitations of noninvasive stimulation methods used for SCI. We further developed a high-resolution finite element method (FEM) model to predict current flow in the spinal cord when using different electrode montages.

\section{Principles of transcutaneous spinal stimulation}

The American Physical Therapy Association has established a unified terminology for electrical currents, namely, direct current (DC), alternating current, and pulsed current. ${ }^{10}$ In DC stimulation, the electric current flows in a constant direction, while in alternating current the direction of flow and amount of electricity change cyclically over time. Alternating current

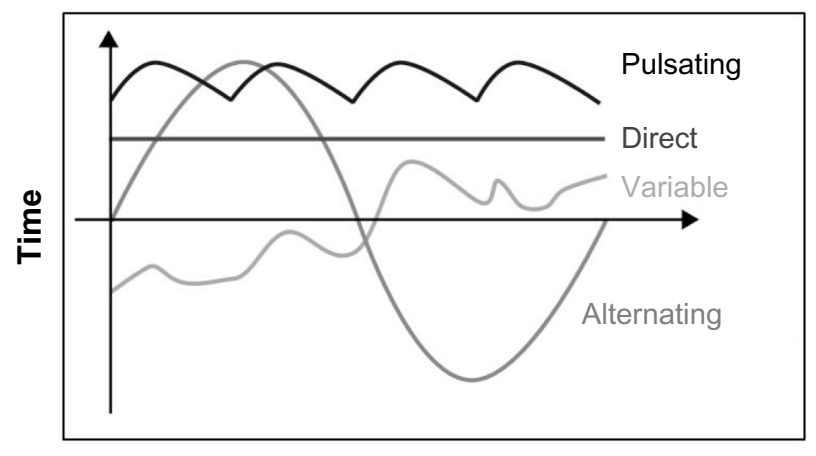

Voltage

Figure I Illustration of various waveforms used for electrical stimulation, including for spinal cord injury.

Notes: Alternating current waveforms can be defined as those that are symmetric in polarity, such as the sinusoid shown. Pulsating, or pulsed, waveforms can be defined as repetitive waveforms characterized by repeated increases in intensity at a given frequency, with broad variations in pulse shape, return phase, frequency, and train rate possible. Still more complex waveforms are possible, which can be defined as being variable. is characterized by a lack of polarity, in other words, there are no defined positive or negative poles. Pulsed or interrupted current is defined as the unidirectional or bidirectional flow of charged particles, with the intensity of the current changing periodically over time. Electric currents may also have different types of wave forms: sinusoidal, rectangular, squared or pointed, depending on the capacity of the generator which produces the current (for details on current, see Robinson ${ }^{11}$ ).

Approaches such as transcutaneous spinal direct current stimulation (tsDCS) use DC and employ a constant intensity for the duration of neuromodulation except for potential ramps at the start and stop of stimulation. Waveforms can be classified as alternating or pulsating, according to their polarity and shape variations. Other complex waveforms are also described. Please see Figure 1 for an illustration of various waveforms used for electrical stimulation.

tsDCS enhances lesion recovery by facilitating the recruitment of inactive motor neurons, ${ }^{12}$ increasing the interaction between functional neuronal circuits, ${ }^{13}$ and suppressing the increased excitability of spinal motor neurons by promoting an inhibitory tone, which in turn can decrease the severity of spasticity following SCI. ${ }^{12}$ The effects vary according to the polarity of stimulation, so that cathodal stimulation activates neurons, whereas anodal stimulation depresses neuronal activity. Furthermore, the first data in healthy subjects suggest that anodal tsDCS may decrease conduction in ascending nociceptive pathways, thereby modulating somatosensory evoked potentials and increasing the pain threshold. ${ }^{14}$ tsDCS is used through a pair of saline-soaked sponge electrodes with a diameter of $35 \mathrm{~cm}^{2}$ and covered with electrolyte gel. The electrical current is delivered by a battery-driven constant current stimulator. Usually one electrode is centered over the spinous process of the vertebra immediately above the targeted area; the other is usually applied over the posterior area of the right shoulder/ limb. Polarity of the stimulation (anodal or cathodal) refers to the polarity of the spinal electrode. Recent publications have proposed protocols using a current intensity of $2.5 \mathrm{~mA}$ during a $15-$ minute period (current density $=0.071 \mathrm{~mA} / \mathrm{cm}^{2}$ - total load $\left.=0.064 \mathrm{C} / \mathrm{cm}^{2}\right) .{ }^{14,15}$ The current is ramped up to the maximal intensity over a period of 10 seconds and similarly ramped down at the end of stimulation. Also, it is suggested that sham stimulation should be set to provide active stimulation for a period of 90 seconds and then stimulation is automatically turned off, in order to simulate the sensation of the actual initial stimulation.

Transcutaneous electric nerve stimulation (TENS) is the application of electrical stimulation through the skin with the main purpose of pain control. ${ }^{16}$ As shown in 


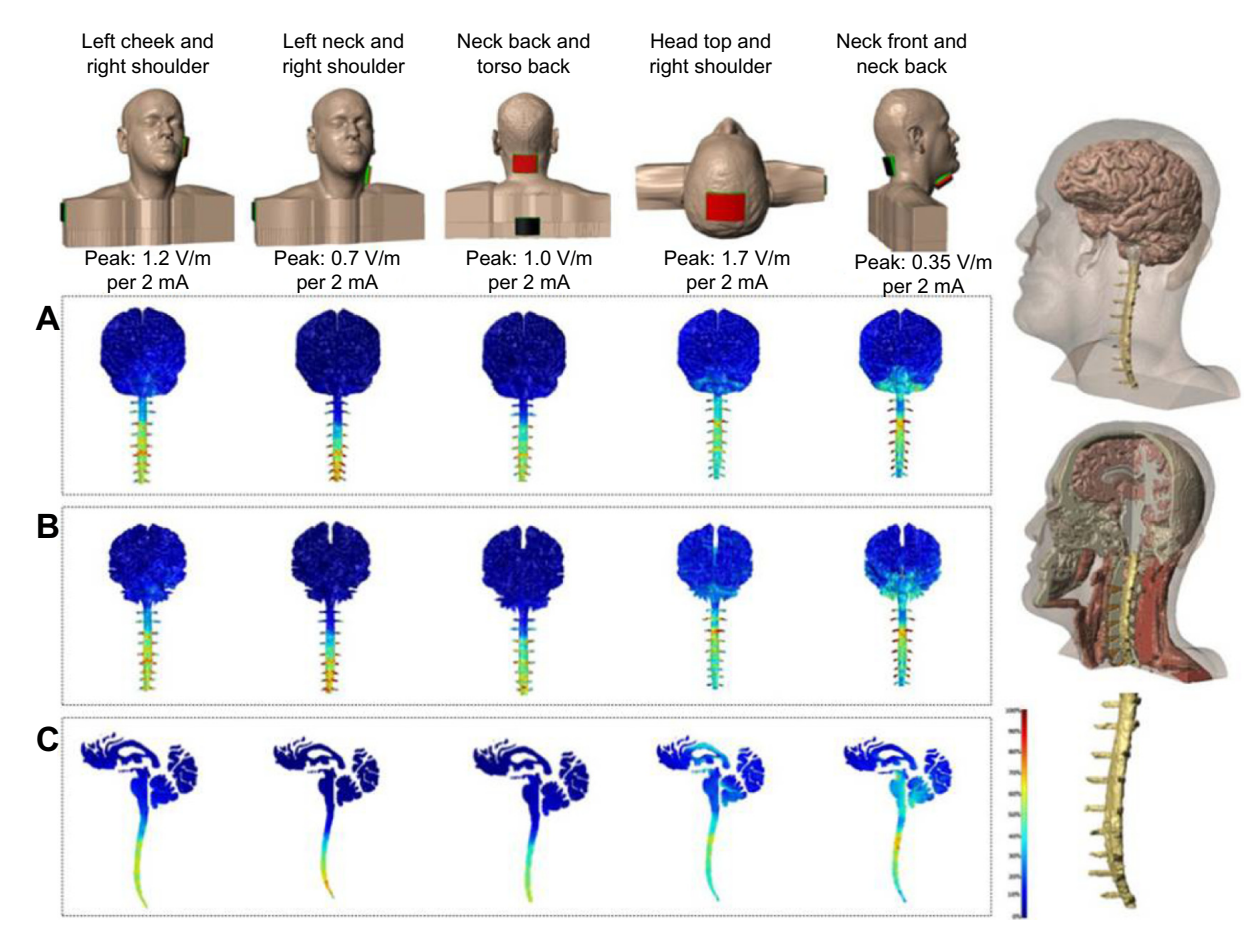

Figure 2 Computational model of current flow across the brain and spinal cord produced by varied noninvasive electrode montages.

Notes: Five electrode montages were simulated (rows) in a magnetic resonance imaging-derived model of one adult. (Top row) Montages used are shown for each column. (Right column) Segmented anatomy of simulation, including a precise representation of the spinal cord. (A) Volumetric plot of the electrical field generated in the gray matter, white matter, and spinal cord. (B) Volumetric plot of the electrical field in the white matter and spinal cord (gray matter removed to reveal underlying tissue). (C) Cross-sectional plot of gray matter, white matter, and spinal cord. In each case, the electrical field is represented in false color. This demonstration shows that significant current reaches the spinal cord during stimulation with extracephalic electrodes, which is consistent with prior modeling efforts and further emphasizes the important of electrode montage in determining current flow and precision in anatomic modeling (eg, spinal root) in simulation accuracy.

Table 1, there are various applications, differing in their parameters. All types have important clinical applications and are recognized as therapeutic approaches available for rehabilitation of SCI, even if the best approach is not fully established. ${ }^{17,18}$ Neuromuscular electrical stimulation (NMES) has been applied as an effective adjunctive therapy to increase muscle strength. ${ }^{19}$ Muscle recruitment is determined by the intensity, duration (loading phase or pulse), and frequency of the stimulus (Table 1). After reaching the motor threshold, a small increase in intensity could produce greater muscle tension, possibly leading to tetanic contraction. Therefore, we should be cautious about increased intensity in order to avoid intense and unwanted contractions. ${ }^{19,20}$ Both pulsed and alternating current are used in NMES devices. ${ }^{21}$

\section{Physiological basis for therapeutic activity of transcutaneous spinal stimulation in SCI Physiological basis for improved motor function and reduced spasticity}

tsDCS is a neuromodulatory technique that leads to direct and long-term changes in the excitability of the spinal cord. ${ }^{22}$ It has been shown that cathodal tsDCS stimulation modulates spinal interneuron excitability to promote motor function. ${ }^{23}$ The activation of the Ia-reciprocal inhibition pathway can reduce spinal plasticity by decreasing the amplitude of the persistent inward currents in spinal motor neurons and the level of excitability of motor neurons. ${ }^{24}$ tsDCS has previously shed light on the modulation of excitability of primary afferent fibers by way of their presynaptic terminals. ${ }^{25}$ Aforementioned evidence provides the principle for clinical application of tsDCS in disorders of maladaptive excitationinhibition balance. Thereby, tsDCS has been applied for the treatment of spasticity. ${ }^{26}$

NMES is used to supplement the lost motor functions and can partially neutralize the harmful adaptations that skeletal muscles endure after SCI. ${ }^{27}$ NMES can activate both motor and sensory axons. The activation of motor neuron axons produces contraction by peripheral pathways, so could generate functional improvement and enhance muscle strength. Activation of sensory axons could also induce muscle contraction by means of signals passing through central pathways. ${ }^{28}$ Consequently, NMES is one of the techniques most commonly used to increase contraction in paralyzed muscles and ameliorate spasticity. 


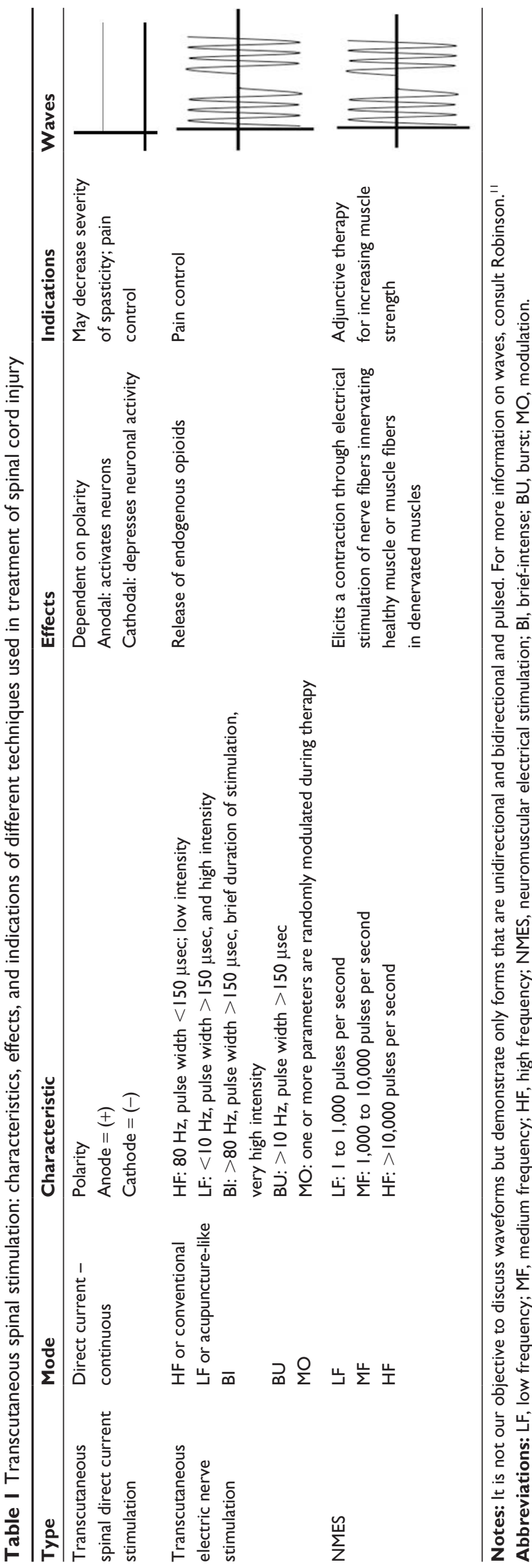

\section{Physiological basis for treatment of neuropathic pain}

tsDCS could interfere with the ascending nociceptive spinal pathway by reducing the abnormal response from spinal circuits after the block in afferent input resulting from the spinal cord lesion. tsDCS could exert this effect by directly activating segmental interneurons (ie, wide dynamic range neurons) or by mediating dorsal column transmission by way of collaterals to the dorsal horns. Alternatively, tsDCS may be able to activate supraspinal loops, transmitted by the brainstem or thalamocortical systems, thereby providing both afferent and efferent inhibition. ${ }^{29} \mathrm{~A}$ review focusing on the physiological effects of this technique and its potential clinical applications suggested that tsDCS could modulate activity in afferent (lemniscal/spinothalamic) and efferent (corticospinal) tracts, as well as in segmental spinal motor circuits. $^{30}$

TENS has been evaluated for SCI-relevant chronic pain in several studies with varied results. Here we discuss two different theoretical mechanisms for TENS using different stimulation frequencies. According to the gate control theory, proposed by Melzack and Wall, ${ }^{31}$ stimulation of large diameter fibers, such as A-beta fibers, would activate inhibitory interneurons in the gelatinous substance of the spinal cord at the dorsal horn, and hence counteract the drive of nociceptive signals from small diameter nerves, eg, A-delta fibers and C fibers. ${ }^{31,32}$ Supraspinal mechanisms relevant to the release of endogenous opioids have also been proposed. ${ }^{33}$ Herein, analgesia induced by low-frequency TENS is usually explained by the release of endogenous opioids in the spinal cord, which could be related to activation of local circuits within the spinal cord or as a result of stimulation of descending inhibitory pathways. ${ }^{9}$

\section{Evidence from animal models of $\mathrm{SCl}$}

Direct stimulation by invasive procedures in the spinal cord was the basis for understanding the application of DC stimulation in the restoration of physiological function. In the early 1940s, experimental animal models of SCI showed that constant current flows longitudinally along the spine in frogs. ${ }^{34}$ In 1951, Marsan et al suggested that inhibition of spinal motor neurons by DC flow in the spinal cord of rats would cause alterations in the rhythmic activity of neurons of the anterior horn. ${ }^{34}$

One study in cats provided evidence related to the mechanisms involved in synaptic potentiation. ${ }^{25} \mathrm{DC}$ was applied in a dorsoventral direction and able to modify both the membrane and the peak potentials of the primary afferent fibers. Dorsal hyperpolarization and ventral depolarization was observed 
when the positive electrode was placed on the back and anterior area, respectively. ${ }^{25}$ Borgens et al found an increase in regeneration of reticulospinal axons in the lamprey when transected fibers faced the cathode of the applied voltage gradient. ${ }^{35}$ Roederer et al determined that calcium influx influenced the degree of degeneration in the proximal segments of the axon because it could affect the stability of the fibrous proteins that comprise the neuronal cytoskeleton. ${ }^{36}$ Corroborating these findings, Strautman et al showed that negative DC flow attenuated entry of intracellular calcium ions into axons. ${ }^{37}$

Some studies have demonstrated that applying DC fields promotes functional recovery in rats following acute SCI. In 1988, Fehlings et al were the first to show that application of DC results in functional improvement in an experimental model of SCI. The motor evoked potential was one of the measurements for evaluation of functional improvement. The results showed an increase in motor evoked potential in the treated group compared with the control group. ${ }^{38}$ Further studies conducted by Borgens et al confirmed these results, suggesting that axons preferentially grow toward a negative charge, facilitated by the imposition of an electric field..$^{39,40}$

Another study investigating the effects of using DC suggested that DC can modulate the activity of the supraspinal somatosensory system. The findings suggested that such modulation depends on the polarity of the stimulation electrode; anodal stimulation increased spontaneous activity and decreased the amplitude of evoked responses in the gracile nucleus, while cathodal stimulation had the opposite effect. The gracile nucleus is the main entry point of the hindpaw somatosensory signals reaching the primary somatosensory cortex in anesthetized rats. ${ }^{41}$

Recently, the use of tsDCS has been studied in animal models in order to investigate physiological changes that support its clinical use. Ahmed et al demonstrated that cathodal tsDCS increases spontaneous activity in the tibial nerve, while anodal decreases the magnitude of muscle contractions. This suggests that the local effects of polarized currents in the spinal cord are complex, and depend on current intensity, duration of stimulation, and type of neural elements involved (cell body or fibers), and also on the distance between the electrodes and anatomic tissues. ${ }^{22}$ Another important observation from the same authors suggested that spinal cord neurons are recruited in an orderly manner. Namely, tsDCS is able to increase the excitability of the spinal cord and maintain a normal recruitment pattern of motor neurons, which is critical to modulate both speed and power circuits. ${ }^{42}$ Ahmed et al also found that tsDCS reduced muscle tone in rats after spinal cord lesioning, which suggests that tsDCS can be a useful tool in the control of spasticity. Spinal-to-sciatic tsDCS reduced transit and steady stretchinduced nerve and muscle responses when compared with spasticity in SCI rats and controls, whereas sciatic-to-spinal tsDCS had opposite effects. ${ }^{43}$

All of these invasive studies have shed light into the mechanisms of tsDCS: tsDCS can selectively modulate the spontaneous activity and intrinsic properties of motor neurons via the somatosensory system; ${ }^{22}$ tsDCS acts as a modulator of spinal excitability, which could be mediated by changes in glutamatergic neurotransmission and can in turn influence cortically elicited muscle contractions in a polarity-dependent fashion; ${ }^{44,45}$ cathodal tsDCS could be used to enhance spinal excitability and to improve function via amplification of cortically evoked movements through spinal mechanisms; ${ }^{23}$ tsDCS could modulate associative plasticity, and combined cortical and peripheral stimulation could produce immediate enhancement of spinal and cortical outputs, respectively, which could in turn improve skilled locomotor recovery after SCI in mice. ${ }^{42}$

Some animal studies have also addressed the use of TENS in SCI. TENS is thought to induce analgesia at the spinal cord level through two different supraspinal pathways, ${ }^{9}$ ie, the rostral ventral medulla/periaqueductal gray in the dorsal midbrain and the medullary reticular nucleus in the spinal cord. Both pathways converge at the level of the spinal cord using similar inhibitory neurotransmitters but can also be activated by similar cortical sites, such as the cingulate cortex. ${ }^{46}$ Further, Clement and Somers suggested that TENS might have a specific effect in the central nervous system depending on stimulation parameters. These authors demonstrated that daily application of TENS diminished mechanical allodynia but not thermal allodynia. They found an increase of gamma aminobutyric acid in the posterior horns bilaterally when used in high-frequency ranges, and an increase in glutamate, aspartate, and glycine bilaterally when a combined technique (low frequency and high frequency) was used. Further studies are essential to assess the behavioral effects, biological effects, and mechanism of action of TENS in SCI. ${ }^{47}$

\section{Finite element method model}

We used FEM models to predict current flow in the brain stem and spinal cord for various extracephalic $1 \times 1$ montages using $5 \times 7 \mathrm{~cm}$ sponge pad electrodes. We developed a high resolution magnetic resonance imaging (MRI) scan $\left(1 \mathrm{~cm}^{3}\right.$ voxels) with eleven tissue compartments. Specific conductivity values obtained from human and rat cell experiments were assigned to the specific tissue compartments between the $\mathrm{C} 7$ vertebra and the vertex. FEM models were generated 
to predict current flow in volume conductor physics studies for multiple electrode montages. Spinal cord and skin masks were artificially extended from the MRI-based masks to prevent current accumulation near boundary limits. Five montages were tested (left cheek to right shoulder, left neck and right shoulder, neck back and torso back, head top and right shoulder, neck front and neck back). Evidently, these representations show a small sample of variations in montages (and factors such as electrode size or number of electrodes are not considered) - our objective here is not to conduct a comprehensive optimization for a myriad of indications, but rather illustrate the principles of how spinal current flow is modulated by electrode montage selection.

Figure 2 shows five electrode montages simulated in an MRI-derived model of one adult. This FEM model demonstrates that significant current is able to reach the spinal cord when extra cephalic electrodes are used for stimulation.

Electric field and current flow clusters were found across compartments of the spinal cord. Notably, small pockets of high current intensity accumulation were found in spinal nerves, which may be attributed to idiosyncrasies in anatomy. These results illustrate that the electrode montage can be designed so that specific spinal segments can be differentially activated and also to determine the direction of current flow, as well as to predict the intensity of the electric field generated per mA of current applied for each montage. The peak electric field per applied $\mathrm{mA}$ in the spinal cord ranged from $0.35 \mathrm{~V} / \mathrm{m}$ (for neck front to neck back) to $1.7 \mathrm{~V} / \mathrm{m}$ (for head top to right shoulder montage). In all cases, the peak electric field was predicted at spinal roots. This concentration represents the anatomy where the spinal cord is surrounded by a highly conductive sheet of cerebrospinal fluid, emphasizing the importance of generating precise models.

\section{Clinical evidence for therapeutic application of transcutaneous spinal stimulation in $\mathbf{S C l}$}

As the field advances, there is increasing interest in conducting human research on this topic focusing on neurophysiology or behavioral clinical effects. Concerning the neurophysiologic parameters, several studies in humans have demonstrated that tsDCS affects the excitability of the spinal cord and induces significant changes in the Hoffman reflex. ${ }^{15,30,48-51}$ The Hoffman reflex is frequently used as a test to assess modulation of spinal inhibitory interneuronal circuits. Changes in excitability of the Hoffman reflex have been related to the acquisition of new motor skills and restoration of motor functions. ${ }^{15}$ Even in healthy individuals, anodal tsDCS was able to decrease the response of the Hoffman reflex while cathodal stimulation led to an increase reaction. tsDCS also causes alterations in somatosensory evoked potentials in healthy subjects. For example, it has been shown that anodal stimulation in the thoracic region depresses the PTN-SEP (32 sequential posterior tibial nerve somatosensory evoked potentials; P-30) component of the posterior tibial nerve. ${ }^{14}$ These results suggest that tsDCS can induce sustained changes in trans-synaptic efficacy in neurons, ${ }^{15}$ which can aid in preventing neuronal dysfunction after SCI. ${ }^{52}$ Despite being one of the oldest and simplest currents used in the field of rehabilitation, tsDCS is now considered a new technique of neuromodulation and there are a few reports using tsDCS after injury, as shown in Table 2.

As noted in Table 2 and discussed above, tsDCS influences conduction along the ascending spinal neural network, reflected by changes in the Hoffman reflex, ${ }^{15,52}$ which represents the monosynaptic connection between Ia muscle spindle fibers and the homonymous innervating motor neuron; the electrical equivalent of the spinal stretch reflex. ${ }^{53}$

With regard to behavioral clinical effects like spasticity and pain relief, we only found one study assessing the therapeutic effects of tsDCS for the relief of spasticity and improvement of motor control after SCI. Hubli et al used tsDCS combined with motor training to establish whether the excitability of the neurons could be modified after complete SCI, by assessing changes in the spinal reflex. They found an $84 \%$ rise in the amplitude of the spinal reflex in subjects with SCI after anodal tsDCS. This effect was not seen for their cathodal tsDCS, locomotion, or sham groups. Moreover, the reflex threshold was lower after anodal tsDCS and locomotion in subjects with SCI. The authors concluded that tsDCS can be repeatedly applied as an auxiliary therapeutic approach for SCI, and that anodal tsDCS can avoid adverse changes in spinal reflex function in severely affected individuals. ${ }^{52}$

Similarly, TENS may reduce spasticity ${ }^{46}$ and improve bladder function in SCI patients. ${ }^{54}$ Hofstoetter et al conducted a pilot study using three subjects with chronic incomplete SCI, and evaluated the effects on reflex excitability and used the $10 \mathrm{~m}$ walk test as a clinical measure. Preliminary results suggest that stimulation can be used to control spasticity without adversely affecting residual motor control in incomplete SCI. ${ }^{55}$ However, this is a pilot study and further studies are needed.

Another important clinical application of this technique is related to pain control. There is a high prevalence of pain in patients with SCI, and many approaches, including pharmacological and nonpharmacological treatments, have 
Table 2 Clinical trials on effects of transcutaneous spinal stimulation in treatment of spinal cord injury

\begin{tabular}{|c|c|c|c|c|c|c|}
\hline Reference & Type & Subjects & Montage & Protocol & Instrument & Result \\
\hline \multirow[t]{6}{*}{ Lamy and Boakye ${ }^{49}$} & $\mathrm{CO}$ & Healthy $(n=17)$ & ESt: Anode SP-I I th & $2.5 \mathrm{~mA}$ & Sol H-reflex & \\
\hline & & & ERf: Cathode shoulder R & 15 minutes & Anode & + \\
\hline & & & & & Cathode & 0 \\
\hline & & & & & BDNF genotyping & \\
\hline & & & & & Anode & + \\
\hline & & & & & Cathode & 0 \\
\hline \multirow[t]{4}{*}{ Cogiamanian et al ${ }^{14}$} & $\mathrm{CO}$ & Healthy $(n=12)$ & ESt: SP-I I th & $2.5 \mathrm{~mA}$ & SEPs & \\
\hline & & & ERf: shoulder R & 15 minutes & Anode & - \\
\hline & & & Sham & & Cathode & + \\
\hline & & & & & Sham & 0 \\
\hline \multirow[t]{3}{*}{ Cogiamanian et $\mathrm{a}^{129}$} & $\mathrm{CO}$ & Healthy $(n=I I)$ & ESt: Anode SP-I I th & $2 \mathrm{~mA}$ & MEPs & \\
\hline & & & ERf: Cathode shoulder R & 15 minutes & Anode & - \\
\hline & & & Sham & & Sham & 0 \\
\hline \multirow[t]{5}{*}{ Hubli et a ${ }^{52}$} & CT & Motor complete SCl $(n=17)$ & ESt: SP-I I th & $2.5 \mathrm{~mA}$ & Spinal reflex & \\
\hline & & Healthy $(n=17)$ & ERf: shoulder R & 20 minutes & Anode: SCl/healthy & $+/ 0$ \\
\hline & & & Sham & & Cathode: SCI/healthy & $0 /-$ \\
\hline & & & & & Sham: SCl/healthy & $0 /-$ \\
\hline & & & & & Locomotion & $0 / 0$ \\
\hline \multirow[t]{6}{*}{ Truini et $\mathrm{a}^{51}$} & $\mathrm{CO}$ & Healthy $=17$ & ESt: SP-I I th & $2.5 \mathrm{~mA}$ & SEPs & \\
\hline & & & ERf: shoulder L & 20 minutes & Anode & - \\
\hline & & & & & Cathode & 0 \\
\hline & & & & & Cold pressor test & \\
\hline & & & & & Anode & - \\
\hline & & & & & Cathode & 0 \\
\hline \multirow[t]{4}{*}{ Winkler et al ${ }^{15}$} & $\mathrm{CO}$ & Healthy $(n=10)$ & ESt: SP-I I th & $2.5 \mathrm{~mA}$ & Sol H-reflex & \\
\hline & & & ERf: shoulder R & 20 minutes & Anode & - \\
\hline & & & Sham & & Cathode & + \\
\hline & & & & & Sham & 0 \\
\hline \multirow[t]{6}{*}{ Yamaguchi et $\mathrm{a}^{50}$} & $\mathrm{CO}$ & Healthy $(n=10)$ & ESt: SP-I I th & $2.5 \mathrm{~mA}$ & Sol H-reflex & \\
\hline & & & ERf: shoulder R & 15 minutes & Anode & - \\
\hline & & & Sham & & Sham & 0 \\
\hline & & & & & SEPs & \\
\hline & & & & & Anode & 0 \\
\hline & & & & & Sham & 0 \\
\hline
\end{tabular}

Notes: 0 , indicates unaltered; +, indicates an increase; -, indicates a decrease.

Abbreviations: BDNF, Brain-derived neurotrophic factor; CO, crossover; CT, controlled trial; SCl, spinal cord injury; ESt, electrode stimulated; ERf, electrode reference; SP, spinous process; Sol H-reflex, soleus H-reflex; SEPs, somatosensory evoked potentials; MEPs, motor evoked potentials; R, right; L, left.

been studied for the treatment of this condition. The results reported by Truini et al suggest that the analgesic effects of tsDCS are a result of the decrease in ascending nociceptive spinal input. One experiment in healthy individuals showed that anodal tsDCS might decrease conduction in the ascending nociceptive spinal network, thus inducing changes in laser evoked potentials in the foot, increasing the pressure threshold ${ }^{51}$ and reducing the nociceptive component of the flexion reflex in the lower limb. ${ }^{29}$ This form of stimulation also alters long-lasting plasticity in spinal motor circuits as it decreases the Hoffman reflex.

There has been one study of the effect of TENS in the treatment of neuropathic pain in patients with SCI. In this study, both low intensity and high intensity TENS had no statistically significant effects on neuropathic pain in patients with SCI. Despite this result, there was an improvement in clinical manifestations, to the extent that patients asked to continue the treatment after the study. The main limitations of this study relate to methodologic issues, such as the lack of a control group, a low number of participants, and the lack of long-term follow-up. ${ }^{56}$

Another study reported by Celik et al used low-frequency TENS to evaluate changes in pain as assessed on a visual analog scale between day 1 and day 12 of treatment and found a statistically significant reduction in pain level in the treatment group. The results of this study showed that low-frequency TENS reduced neuropathic pain intensity in SCI patients during the daytime but not at night. This can be explained by the fact that neuropathic pain intensity is higher during the night. ${ }^{57}$ This study only included patients with neuropathic pain at or below the level of injury, and is consistent with a study by Störmer et al which reported 
that $86 \%$ of the pain is localized below the level of the SCI lesion. ${ }^{58}$

Fishbain et al demonstrated that TENS has long-term effects on quality of life in patients with chronic pain, in particular the ability to resume daily activities. It also showed an increased level of pain management and lower use of drugs and other therapies. ${ }^{59}$ Therefore, it can be useful to assess the effects of long-term TENS in future studies.

\section{Current status: utility, limitations, and potential refinements}

This review highlights the limited number of controlled studies addressing transcutaneous spinal stimulation for the treatment of SCI patients. This can be attributed to a lack of knowledge of this application or even to the diversity of types of electrical current that can be used in clinical practice. This new treatment modality has emerged in the rehabilitation environment as a useful tool to help in the rehabilitation process of different clinical conditions. However, there is a need for further large-scale studies to confirm the benefits and risks of this resource on patients with SCI.

It is known that individuals with an incomplete lesion, who maintain some ability to walk, would likely avail themselves of a walking program that includes body weight support, treadmill training, and NMES. ${ }^{60}$ The results of the combined approach using physical therapy and neuromodulation techniques has led to further consideration of use of tsDCS with conventional or robotic therapy in the treatment of individuals with SCI.

More generally, it is important to study the efficacy of noninvasive electric stimulation in the modulation of spinal networks in patients with SCI to: probe the value of noninvasive electrical stimulation in modifying other pathways in patients suffering from various spinal cord lesions, and estimate the clinical relevance of noninvasive electric stimulation (in combination with other treatment methods or not) as a therapeutic protocol. This therapeutic approach should be considered as an additional nonpharmacological treatment option in an existing treatment plan. For instance, tsDCS is proven to be a simple technique with low operating costs. Nevertheless, a larger randomized controlled trial must be conducted in order to demonstrate its therapeutic potential.

\section{Conclusion}

This review discusses the rationale and mechanisms for noninvasive neurostimulation methods and outlines recent trials on clinical efficacy of transcutaneous spinal stimulation for SCI. According to our review of the existing literature on the use of this neuromodulation technique for the treatment of SCI and as demonstrated by several clinical trials cited in this review (Table 2), transcutaneous spinal stimulation is a promising therapeutic tool for patients suffering from this condition. Although more studies are needed to clarify its therapeutic potential, its efficacy depends on several factors concerning both the individual technique applied and the patient's condition, as follows:

1. The ability of tsDCS to influence conduction along ascending tracts in the spinal cord could help modulate supraspinal activity and also lead to focal changes in spinal cord excitability by DC polarization.

2. TENS has been studied for chronic pain related to SCI in several trials. The development and application of high-frequency TENS was based on the gate control theory of pain conceptualized by Melzack and Wall. ${ }^{32,61,62}$ Although this therapy could be used as an alternative method to deal with chronic pain in SCI patients, results are still inconclusive, so development of novel parameters of stimulation may be necessary to optimize the results of this technique.

3. NMES is one of the most used techniques to improve contraction strength in paralyzed muscles and ameliorate spasticity. This technique is used in rehabilitation and can counterbalance the deleterious adaptations that skeletal muscle undergoes after a lesion to the spinal cord. ${ }^{27}$

4. FEM methods confirm that significant amounts of electrical current are induced in the spinal cord structures when using extracephalic electrodes.

Promoting plasticity with electrical stimulation has been increasingly pursued over recent years. If the results of spinal cord stimulation are confirmed in larger mechanistic and clinical trials, then this potential therapy may be a relevant therapeutic approach for a number of neuropsychiatric conditions.

\section{Acknowledgments}

This study was supported by grants from the Department of Education (NIDRR H133N110010; SCI Model Systems), National Natural Science Foundation of China (81000852 and 81301677), Supporting Project of Science and Technology of Sichuan Province (2012SZ0140) and the Research Foundation of Zhejiang Province (201022896). To CAPES (Coordenadoria de Aperfeicoamento de Pessoal do Ensino Superior) for the scholarship granted to LHG (University of São Paulo). The authors acknowledge Leon Morales-Quezada and Danuza Nunn for their assistance with editing this review. 


\section{Disclosure}

The authors report no conflicts of interest in this work.

\section{References}

1. Rossignol S, Schwab M, Schwartz M, Fehlings MG. Spinal cord injury: time to move? J Neurosci. 2007;27(44):11782-11792.

2. Cripps R, Lee B, Wing P, Weerts E, Mackay J, Brown D. A global map for traumatic spinal cord injury epidemiology: towards a living data repository for injury prevention. Spinal Cord. 2011;49(4):493-501.

3. Krause JS. Risk for subsequent injuries after spinal cord injury: a 10-year longitudinal analysis. Arch Phys Med Rehabil. 2010;91(11): 1741-1746.

4. Krause JS, Zhai Y, Saunders LL, Carter RE. Risk of mortality after spinal cord injury: an 8-year prospective study. Arch Phys Med Rehabil. 2009;90(10):1708-1715.

5. Barriere G, Leblond H, Provencher J, Rossignol S. Prominent role of the spinal central pattern generator in the recovery of locomotion after partial spinal cord injuries. J Neurosci. 2008;28(15):3976-3987.

6. Pinter MM, Dimitrijevic MR. Gait after spinal cord injury and the central pattern generator for locomotion. Spinal Cord. 1999;37(8):531-537.

7. Frigon A, Rossignol S. Functional plasticity following spinal cord lesions. Prog Brain Res. 2006;157:231-260.

8. Schuhfried O, Crevenna R, Fialka-Moser V, Paternostro-Sluga T. Non-invasive neuromuscular electrical stimulation in patients with central nervous system lesions: an educational review. $J$ Rehabil Med. 2012;44(2):99-105.

9. Sluka KA, Deacon M, Stibal A, Strissel S, Terpstra A. Spinal blockade of opioid receptors prevents the analgesia produced by TENS in arthritic rats. J Pharmacol Exp Ther. 1999;289(2):840-846.

10. Alon G. Electrotherapeutic Terminology in Physical Therapy: Apta Section on Clinical Electrophysiology. Alexandria, VA, USA: American Physical Therapy Association; 2005.

11. Robinson AJ. Clinical Electrophysiology: Electrotherapy and Electrophysiologic Testing. Lippincott Williams \& Wilkins; 2008.

12. Elbasiouny SM, Mushahwar VK. Suppressing the excitability of spinal motoneurons by extracellularly applied electrical fields: insights from computer simulations. J Appl Physiol. 2007;103(5):1824-1836.

13. Radman T, Su Y, An JH, Parra LC, Bikson M. Spike timing amplifies the effect of electric fields on neurons: implications for endogenous field effects. J Neurosci. 2007;27(11):3030-3036.

14. Cogiamanian F, Vergari M, Pulecchi F, Marceglia S, Priori A. Effect of spinal transcutaneous direct current stimulation on somatosensory evoked potentials in humans. Clin Neurophysiol. 2008;119(11):2636-2640.

15. Winkler T, Hering P, Straube A. Spinal DC stimulation in humans modulates post-activation depression of the H-reflex depending on current polarity. Clin Neurophysiol. 2010;121(6):957-961.

16. American Physical Therapy Association. Guide to Physical Therapist Practice. Second edition. American Physical Therapy Association. Phys Ther. 2001;81(1):9-746.

17. Bélanger A, Bélanger A. Therapeutic Electrophysical Agents: Evidence Behind Practice. 2nd ed. Philadelphia, PA, USA: Wolters Kluwer Health/Lippincott Williams \& Wilkins; 2010.

18. Johnson MI, Ashton CH, Thompson JW. The consistency of pulse frequencies and pulse patterns of transcutaneous electrical nerve stimulation (TENS) used by chronic pain patients. Pain. 1991;44(3):231-234.

19. Lewek M, Stevens J, Snyder-Mackler L. The use of electrical stimulation to increase quadriceps femoris muscle force in an elderly patient following a total knee arthroplasty. Phys Ther. 2001;81(9):1565-1571.

20. Caggiano E, Emrey T, Shirley S, Craik RL. Effects of electrical stimulation or voluntary contraction for strengthening the quadriceps femoris muscles in an aged male population. J Orthop Sports Phys Ther. 1994;20(1):22-28

21. Ward AR, Oliver WG, Buccella D. Wrist extensor torque production and discomfort associated with low-frequency and burst-modulated kilohertz-frequency currents. Phys Ther. 2006;86(10):1360-1367.
22. Ahmed Z. Trans-spinal direct current stimulation modulates motor cortex-induced muscle contraction in mice. JAppl Physiol. 2011;110(5): 1414-1424.

23. Ahmed Z. Effects of cathodal trans-spinal direct current stimulation on mouse spinal network and complex multijoint movements. J Neurosci. 2013;33(37):14949-14957.

24. Kuo JJ, Lee RH, Johnson MD, Heckman HM, Heckman CJ. Active dendritic integration of inhibitory synaptic inputs in vivo. J Neurophysiol. 2003;90(6):3617-3624.

25. Eccles JC, Kostyuk PG, Schmidt RF. The effect of electric polarization of the spinal cord on central afferent fibres and on their excitatory synaptic action. J Physiol. 1962;162:138-150.

26. Elbasiouny SM, Moroz D, Bakr MM, Mushahwar VK. Management of spasticity after spinal cord injury: current techniques and future directions. Neurorehabil Neural Repair. 2010;24(1):23-33.

27. Shields RK, Dudley-Javoroski S. Musculoskeletal plasticity after acute spinal cord injury: effects of long-term neuromuscular electrical stimulation training. $J$ Neurophysiol. 2006;95(4):2380-2390.

28. Bergquist AJ, Clair JM, Lagerquist O, Mang CS, Okuma Y, Collins DF. Neuromuscular electrical stimulation: implications of the electrically evoked sensory volley. Eur J Appl Physiol. 2011;111(10): 2409-2426.

29. Cogiamanian F, Vergari M, Schiaffi E, et al. Transcutaneous spinal cord direct current stimulation inhibits the lower limb nociceptive flexion reflex in human beings. Pain. 2011;152(2):370-375.

30. Cogiamanian F, Ardolino G, Vergari M, et al. Transcutaneous spinal direct current stimulation. Front Psychiatry. 2012;3:63.

31. Melzack R, Wall PD. Pain mechanisms: a new theory. Science. 1965 150(3699):971-979.

32. Melzack R, Vetere P, Finch L. Transcutaneous electrical nerve stimulation for low back pain. A comparison of TENS and massage for pain and range of motion. Phys Ther. 1983;63(4):489-493.

33. Han JS, Chen XH, Sun SL, et al. Effect of low- and high-frequency TENS on Met-enkephalin-Arg-Phe and dynorphin A immunoreactivity in human lumbar CSF. Pain. 1991;47(3):295-298.

34. Marsan CA, Fuortes M, Marossero F. Effects of direct currents on the electrical activity of the spinal cord. $J$ Physiol. 1951;113(2-3): 316-321.

35. Borgens RB, Roederer E, Cohen MJ. Enhanced spinal cord regeneration in lamprey by applied electric fields. Science. 1981;213(4508): 611-617.

36. Roederer E, Goldberg NH, Cohen MJ. Modification of retrograde degeneration in transected spinal axons of the lamprey by applied DC current. J Neurosci. 1983;3(1):153-160.

37. Strautman AF, Cork RJ, Robinson KR. The distribution of free calcium in transected spinal axons and its modulation by applied electrical fields. J Neurosci. 1990;10(11):3564-3575.

38. Fehlings MG, Tator $\mathrm{CH}$, Linden $\mathrm{RD}$. The effect of direct-current field on recovery from experimental spinal cord injury. J Neurosurg. 1988;68(5):781-792.

39. Borgens R. Electrically mediated regeneration and guidance of adult mammalian spinal axons into polymeric channels. Neuroscience. 1999;91(1):251-264.

40. Borgens RB, Bohnert DM. The responses of mammalian spinal axons to an applied DC voltage gradient. Exp Neurol. 1997;145(2): 376-389.

41. Aguilar J, Pulecchi F, Dilena R, Oliviero A, Priori A, Foffani G. Spinal direct current stimulation modulates the activity of gracile nucleus and primary somatosensory cortex in anaesthetized rats. $J$ Physiol. 2011;589(20):4981-4996.

42. Ahmed Z. Electrophysiological characterization of spino-sciatic and cortico-sciatic associative plasticity: modulation by trans-spinal direct current and effects on recovery after spinal cord injury in mice. J Neurosci. 2013;33(11):4935-4946.

43. Ahmed Z. Trans-spinal direct current stimulation alters muscle tone in mice with and without spinal cord injury with spasticity. J Neurosci. 2014;34(5):1701-1709. 
44. Ahmed Z, Wieraszko A. Trans-spinal direct current enhances corticospinal output and stimulation-evoked release of glutamate analog, D-2, 3-3H-aspartic acid. J Appl Physiol. 2012;112(9):1576-1592.

45. Camchong J, MacDonald AW III, Bell C, Mueller BA, Lim KO. Altered functional and anatomical connectivity in schizophrenia. Schizophr Bull. 2009;2009:sbp131.

46. Liebano R, Vance C, Rakel B, et al. Transcutaneous electrical nerve stimulation and conditioned pain modulation influence the perception of pain in humans. Eur J Pain. 2013;17(10):1539-1546.

47. Somers DL, Clemente FR. Contralateral high or a combination of highand low-frequency transcutaneous electrical nerve stimulation reduces mechanical allodynia and alters dorsal horn neurotransmitter content in neuropathic rats. J Pain. 2009;10(2):221-229.

48. Lamy JC, Ho C, Badel A, Arrigo RT, Boakye M. Modulation of soleus H reflex by spinal DC stimulation in humans. J Neurophysiol. 2012;108(3):906-914.

49. Lamy JC, Boakye M. Seeking significance for transcutaneous spinal DC stimulation. Clin Neurophysiol. 2013;124(6):1049-1050.

50. Yamaguchi T, Fujimoto S, Otaka Y, Tanaka S. Effects of transcutaneous spinal DC stimulation on plasticity of the spinal circuits and corticospinal tracts in humans. Paper presented at the 6th International IEEE/ EMBS Conference on Neural Engineering. November 6-8, 2013, San Diego, CA, USA.

51. Truini A, Vergari M, Biasiotta A, et al. Transcutaneous spinal direct current stimulation inhibits nociceptive spinal pathway conduction and increases pain tolerance in humans. Eur J Pain. 2011;15(10): 1023-1027.

52. Hubli M, Dietz V, Schrafl-Altermatt M, Bolliger M. Modulation of spinal neuronal excitability by spinal direct currents and locomotion after spinal cord injury. Clin Neurophysiol. 2013;124(6):1187-1195.

53. DeBruin H, Fu W, Galea V, McComas A. Speculations surrounding a spinal reflex. J Neurol Sci. 2006;242(1):75-82.
54. Radziszewski K, Zielinski H, Radziszewski P, Swiecicki R. Transcutaneous electrical stimulation of urinary bladder in patients with spinal cord injuries. Int Urol Nephrol. 2009;41(3):497-503.

55. Hofstoetter US, McKay WB, Tansey KE, Mayr W, Kern H, Minassian K. Modification of spasticity by transcutaneous spinal cord stimulation in individuals with incomplete spinal cord injury. J Spinal Cord Med. 2014;37(2):202-211.

56. Norrbrink C. Transcutaneous electrical nerve stimulation for treatment of spinal cord injury neuropathic pain. J Rehabil Res Dev. 2009;46(1): 85-93.

57. Celik E, Erhan B, Gunduz B, Lakse E. The effect of low-frequency TENS in the treatment of neuropathic pain in patients with spinal cord injury. Spinal Cord. 2013;51(4):334-337.

58. Störmer S, Gerner H, Grüninger W, et al. Chronic pain/dysaesthesiae in spinal cord injury patients: results of a multicentre study. Spinal Cord. 1997;35(7):446-455.

59. Fishbain DA, Chabal C, Abbott A, Heine LW, Cutler R. Transcutaneous electrical nerve stimulation (TENS) treatment outcome in long-term users. Clin J Pain. 1996;12(3):201-214.

60. Field-Fote EC. Combined use of body weight support, functional electric stimulation, and treadmill training to improve walking ability in individuals with chronic incomplete spinal cord injury. Arch Phys Med Rehabil. 2001;82(6):818-824.

61. Melzack R, Vetere P, Finch L. Transcutaneous electrical nerve stimulation for low back pain: a comparison of TENS and massage for pain and range of motion. Phys Ther. 1983;63(4):489-493.

62. Melzack R, Wall PD. Pain mechanisms: a new theory. Survey of Anesthesiology. 1967;11(2):89-90.

\section{Publish your work in this journal}

The Journal of Neurorestoratology is an international, peer-reviewed, open access online journal publishing original research and review articles on the subject of Neurorestoratology. To provide complete coverage of this revolutionary field the Journal of Neurorestoratology will report on relevant experimental research, technological advances, and

\section{Dovepress}

clinical achievements. The manuscript management system is completely online and includes a very quick and fair peer-review system, which is all easy to use. Visit http://www.dovepress.com/testimonials.php to read real quotes from published authors.

Submit your manuscript here: http://www.dovepress.com/journal-of-neurorestoratology-journal 